\title{
Revealing Contact Interval Patterns in Large Scale Urban Vehicular Ad Hoc Networks
}

\author{
Yong Li \\ Electronic Engineering Dept. \\ Tsinghua University \\ Beijing 100084, China \\ liyong07@tsinghua.edu.cn \\ Li Su \\ Electronic Engineering Dept. \\ Tsinghua University \\ Beijing 100084, China \\ lisu@tsinghua.edu.cn
}

\author{
Depeng Jin \\ Electronic Engineering Dept. \\ Tsinghua University \\ Beijing 100084, China \\ jindp@tsinghua.edu.cn
}

\author{
Pan Hui \\ Deutsche Telekom Lab. \\ TU-Berlin \\ Berlin 10587 \\ Pan.Hui@cl.cam.ac.uk
}

\author{
Lieguang Zeng \\ Electronic Engineering Dept. \\ Tsinghua University \\ Beijing 100084, China \\ zenglg@tsinghua.edu.cn
}

\begin{abstract}
Contact interval between moving vehicles is one of the key metrics in vehicular ad hoc networks (VANETs), which is important to routing schemes and network capacity. In this work, by carrying out an extensive experiment involving tens of thousands of operational taxis in Beijing city, we find an invariant character that the contact interval can be modeled by a three-segmented distribution, and there exists a characteristic time point, up to which the contact interval obeys a power law distribution, while beyond which it decays as an exponential one. This property is in sharp contrast to the recent empirical data studies based on Shanghai vehicular mobility, where the contact interval exhibits only exponential distribution.
\end{abstract}

\section{Categories and Subject Descriptors}

C.2.0 [Computer-Communication Networks]: General; C.4 [Performance of Systems]: [Modeling techniques, Performance attributes]

\section{Keywords}

Vehicular networks, contact interval patterns, mobility trace

\section{INTRODUCTION}

Urban vehicular ad hoc networks (VANETs) are recognized as an important element of the future intelligent transportation system to alleviate serious problems, such as traffic jams and accidents, as well as to enable new mobile applications to the public [1]. Since urban VANETs are highly mobile and sometimes sparse, it is hard to maintain a connected network to communicate. In order to transfer data from the source to the destination, vehicles need to first wait until they meet some other vehicles, i.e. until they are within the communication ranges of other vehicles. The performance of these networks, which are based on such type of data transmission, depend strongly on the vehicular mobility characteristics, especially on how often such communication opportunities occurs and on how long they last. In this work,

Copyright is held by the author/owner(s). SIGCOMM'12, August 13-17, 2012, Helsinki, Finland. ACM 978-1-4503-1419-0/12/08. we focus on the metric of contact interval, which is the time interval between two contacts. It is one of the most important factors directly affecting the amount of data that can be transferred between vehicles when they opportunisticly encounter each other. Therefore, revealing its fundamental properties, which hold in large scale vehicular environment, can provide important hints on the characteristics of mobile nodes behaviors in VANETs, and would greatly benefit the applications design like data dissemination schemes in such network environment.

Until now, there exists no credible model reported on the urban vehicular environment based on large scale realistic vehicular mobility trace. Consequently, the current proposed forwarding protocols can only be investigated based on the results obtained from human mobility [3], which may lead to either over-pessimistic or over-optimistic results in the vehicular environment due to the significant differences between human and vehicular mobilities. Therefore, it is in pressing demand to have a profound understanding of practical constraints imposed by the contact interval on opportunistic vehicular data transmission.

However, contact intervals between highly mobile vehicles are usually casual, and existing most largest vehicular trace of Shanghai [2] includes 2,019 operational taxis, based on which Ref. [2] reveals the exponential distribution of contact interval. Since this trace only involves about two thousands of vehicles, it is not enough for studying larger scale vehicular system in modern urban environment which may include tens of millions vehicles, and therefore the obtained result may be not very credible and should vary from different cities. Consequently, a large data record is required to obtain an objective characterization. Due to the costly investment for such experiments, there is no further work studying the contact interval distribution in a realistic large scale urban environment to the best of our knowledge.

\section{DATA COLLECTION}

In collecting Beijing trace, we used the mobility track logs obtained from 27,000 participating Beijing taxis carrying GPS receivers and GPRS module shown in Fig. 1 (a) during May of 2010. The reason for us to choose taxis as vehicular devices is that they are more sensitive to urban environments in terms of underlying road topology, traffic control and urban planning, and they have broader cover- 


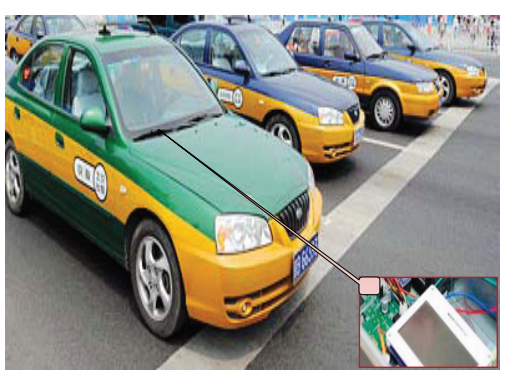

(a)

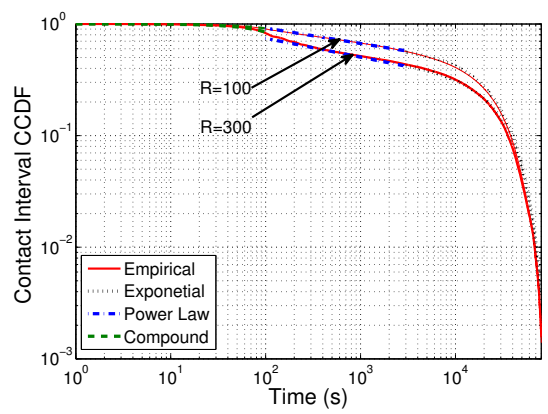

(b)

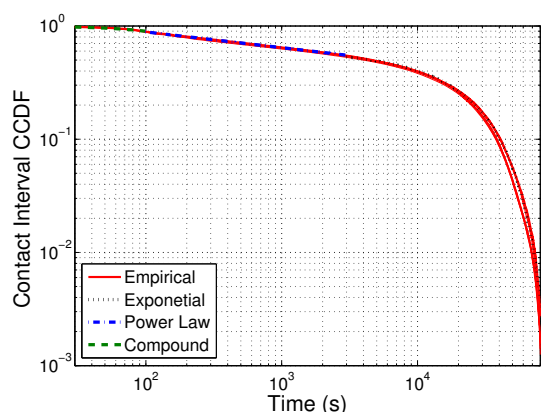

(c)

Figure 1: (a) The illustration of deployed vehicular mobility trace collection system. (b)CCDF of contact interval for the whole month; and (c) CCDF of contact interval for each day in a week $(R=200)$.

age in terms of space and operation time than buses and private cars. Specifically, we utilized the GPS devices to collect the taxis locations and timestamps and GPRS modules to report the records every 15 seconds for moving taxis. The specific information contained in such a report includes: the taxi's ID, the longitude and latitude coordinates of the taxi's location, timestamps, instant speed and heading. Our collected Beijing trace is the largest vehicular data trace available.

\section{MAIN RESULT}

To obtain an accurate contact information, we use the method of linear interpolation to extract the exact beginning and ending times of each contact. The aggregated empirical distributions of contact interval between vehicles were examined, and the obtained CCDFs of contact interval, i.e., $P(X>t)$, are plotted using log-log scale in Fig. 1, where Fig. 1(b) present the aggregate contact intervals of all vehicles under different communication ranges of $R=100$ and $R=300$ during the whole trace collection time while Fig. 1(c) presents the distribution of aggregate contact interval in the time scale of one day under the communications range of $R=200$. By observing the distribution property, we divide the curves by three segments as follows, $[0-100] \mathrm{s}$ , $[100-3000] s$, and $[3000-60000] s$. We use the compound function of $y=b * e^{x^{a}}$ to fit the first part, and use power law and exponential distribution to fit the middle and tail parts respectively. From the empirical distributions of contact intervals that are plotted by the red curves, it can be observed that between the middle and tail parts, there is a "knee" in the distribution plot, which is referred to as the characteristic time. We observe that, the contact interval exhibits a power low distribution before the characteristic time and an exponential tail after the characteristic time, which is in sharp contrast to the results in Ref. [2].

In order to verify the accuracy of the three-segmented distribution model for the contact interval of urban VANETs, we use the respective distributions to fit the first, middle

Table 1: The adjusted R-square statistics of the exponential, power law and compound fittings.

\begin{tabular}{|c|ccc|}
\hline Transmission range & $R=100$ & $R=200$ & $R=300$ \\
\hline Exponential Part & $99.61 \%$ & $99.99 \%$ & $99.99 \%$ \\
Power law Part & $99.32 \%$ & $99.69 \%$ & $99.46 \%$ \\
Compound Part & $99.29 \%$ & $99.41 \%$ & $99.68 \%$ \\
\hline
\end{tabular}

and tail parts, partitioned by the characteristic time, of all the empirical curves in the two figures, respectively. The goodness of fit is measured quantitatively by the R-square statistics, shown in Table 1. It can be seen from Table 1 that the average adjusted R-square statistics is all over $99 \%$ for the three parts. This confirms the accuracy of the threesegmented distribution model for contact interval.

\section{DISCUSSION AND FUTURE WORK}

Different from the existing results found in Shanghai vehicular mobility, in our studies, the first and the second part of contact intervals do not obeys exponential distribution, and only the last tail part obeys the exponential distribution. Our future work will address the fundamental questions of what are the essential factors that generates the three-segmented distribution behavior of the contact interval of taxis as well as how to utilize the three-segmented distribution based contact interval model for helping the routing and forwarding protocol design in urban VANETs.

\section{ACKNOWLEDGMENT}

This work is supported by National Nature Science Foundation of China (No. 61171065, No. 61021001) and Chinese National Major Scientific and Technological Specialized Project (No. 2010ZX03004-002-02), as well as Changjiang Scholars and Innovative Research Team in University (PCSIRT) and Tsinghua National Laboratory for Information Science and Technology (TNLIST).

\section{REFERENCES}

[1] M. Khabazian, S. Aissa, and M. Mehmet-Ali, "Performance modeling of message dissemination in vehicular ad hoc networks with priority," IEEE J. Selected Areas in Communications, vol. 29, no. 1, pp. 61-71, Jan. 2011.

[2] H. Zhu, M. Li, L. Fu, G. Xue, Y. Zhu, and L. Ni, "Impact of traffic influxes: Revealing exponential inter-contact time in urban VANETs," IEEE Trans. Parallel and Distributed Systems, vol. 22, no. 8, pp. 1258-1266, Aug. 2011.

[3] Y. Li, Z. Wang, D. Jin, L. Zeng, and S. Chen, "Collaborative Vehicular Content Dissemination with Directional Antennas," IEEE Transactions on Wireless Communications, vol. 11, no. 4, pp. 1301-1306, April 2012. 